\title{
A systematic review and meta-analysis of capsular contracture rate after breast augmentation with textured and smooth breast prostheses
}

\author{
Peiming Zhai ${ }^{1 \#}$, Yigong $\mathrm{Wu}^{1 \#}$, Deli Yang ${ }^{2}$, Wenhua $\mathrm{Ma}^{2}$, Yabin Zhai ${ }^{1}$, Hongju Xie ${ }^{3}$ \\ ${ }^{1}$ Department of Plastic Surgery, The Fifth People's Hospital of Hainan Province, Haikou, China; ${ }^{2}$ Department of Surgical Anesthesia, The Fifth \\ People's Hospital of Hainan Province, Haikou, China; ${ }^{3}$ Department of Plastic Surgery, The Second Affiliated Hospital of Hainan Medical University, \\ Haikou, China \\ Contributions: (I) Conception and design: P Zhai; (II) Administrative support: Y Wu; (III) Provision of study materials or patients: D Yang; (IV) \\ Collection and assembly of data: W Ma, H Xie; (V) Data analysis and interpretation: P Zhai, Y Zhai; (VI) Manuscript writing: All authors; (VII) Final \\ approval of manuscript: All authors. \\ "These authors contributed equally to this work. \\ Correspondence to: Hongju Xie. Department of Plastic Surgery, The Second Affiliated Hospital of Hainan Medical University, Haikou 570100, China. \\ Email: xiehongju11@163.com.
}

Backgroundk With the development of breast augmentation and the increased people's pursuit of beauty, more and more women choose breast prosthesis breast augmentation. This study aimed to evaluate the incidence of capsular contracture after breast augmentation with textured and smooth breast prostheses by meta-analysis.

Methods: Studies relating to capsular contracture after breast augmentation with textured breast prosthesis and smooth breast prosthesis were retrieved from the Chinese Journal Full-text Database (CNKI), VIP, the Wanfang Science and Technology Journal Full-text Database, the Chinese Biomedical Literature Search Database (CBM), PubMed, Embase, and the Cochrane Library. The search period was from database establishment to October 2021. EndNote X9 software (Clarivate, Philadelphia, PA, USA) was used to screen and exclude duplicate studies, screen the studies according to the inclusion and exclusion criteria, and extract data for quality evaluation. The data were recorded using the Stata 15.1 software (StataCorp LLC, College Station, TX, USA), and a meta-analysis was performed on the incidence of capsular contracture after breast augmentation with textured and smooth breast prostheses. Finally, the reliability of the results was assessed by sensitivity analysis. A funnel plot was used to evaluate the publication bias of the literature.

Results: A total of 13 articles were included, and the total sample size of the study was 5,440. The results of the meta-analysis showed that the incidence of capsular contracture after breast augmentation with textured breast prostheses was significantly lower than that with smooth breast prostheses (OR $=0.26 ; 95 \%$ CI: 0.08 , $0.81 ; \mathrm{P}<0.05)$.

Discussion: The incidence of capsular contracture after breast augmentation with textured breast prosthesis is significantly lower than that with smooth breast prosthesis.

Keywords: Breast prosthesis; breast augmentation; capsular contracture; meta-analysis

Submitted Nov 12, 2021. Accepted for publication Dec 30, 2021.

doi: $10.21037 /$ gs-21-835

View this article at: https://dx.doi.org/10.21037/gs-21-835 


\section{Introduction}

With the progress and development of human society, the breast is no longer simply a lactating organ, but also a characteristic of feminine physical beauty (1). Breast hypoplasia or breast atrophy due to an acquired cause can affect the physical beauty of women and lead to the development of an inferiority complex. Breast augmentation surgery, which has grown more popular in recent years, can quickly restore a woman's beauty and enhance pride and self-confidence.

As early as the late 19th century, surgeons were experimenting with breast augmentation. The earliest record of breast augmentation surgery was in 1895, when Vincenz Czerny transplanted lipomatous tissue from a patient's back into her breast following the resection of a breast adenoma. In 1899, Robert Gersuny opened the way to augmentation with allogeneic materials by directly injecting liquid paraffin into the breast to achieve augmentation. However, this method resulted in inflammatory reactions, poor breast shape, hard nodules in the breast, or paraffin embolism, which could cause blindness or death. After the 1940s, Americans used a mixture of honey and wax for breast augmentation surgery, though this method also had serious complications such as induration, embolism, and inflammatory reactions. During World War II, a method of breast augmentation using silicone injection was popular among Japanese geisha; however, this method caused serious complications such as infection, tumor-like lumps, and chronic inflammation, resulting in mastectomy and serious damage to body organs and ultimately leading to its withdrawal from mainstream medicine.

Since Cronin et al. first used a silicone rubber sac prosthesis in breast augmentation surgery, prosthesis breast augmentation has been generally regarded as a safe and effective method (2). The advantages of this method include reliable materials, good desirability, a reliable effect, and a wide range of application, etc. The continuous improvement and optimization of prosthesis materials and processes has led to the development of different types of breast prostheses. Those commonly used at present include raft-textured double-layer prosthesis, textured saline-filled prosthesis, textured silicone gel prosthesis, smooth salinefilled prosthesis, and smooth silicone gel prosthesis.

Complications of breast augmentation surgery are bleeding/hematoma, infection, prosthetic displacement, rupture, leakage, fiber envelope contracture, psychological disorders, etc. Capsular contracture can lead to poor effects such as the development of a small cyst cavity where the prosthesis is located, the deformation of the compression prosthesis, and a change of shape in the breast, which can result in breast hardening and pain. The present view is that the occurrence of envelope contracture involves biofilm formation caused by bacterial infection and a series of immune responses, and the process involves the participation of various inflammatory cells, cytokines, etc. Breast prostheses can be divided into 2 types: those with a smooth surface and those with a textured surface. The choice of preoperative breast prosthesis mainly includes three aspects: one is to choose the type, two is to choose the appropriate size, three is to check the quality. Postoperative breast shape, position, and hand feel can evaluate the results of breast augmentation. Despite an increase in relevant studies over the past few years, there is still a lack of consensus on the appropriate selection of prosthesis type. This study was analyzed by incorporating the latest studies, using the method of meta-analysis to address this issue by investigating the incidence of capsular contracture after breast augmentation with smooth and textured breast prostheses.

We present the following article in accordance with the PRISMA reporting checklist (available at https:// gs.amegroups.com/article/view/10.21037/gs-21-835/rc).

\section{Methods}

\section{Literature search strategy}

Literature was retrieved from foreign major journal databases including the Chinese Journal Full-text Database (CNKI), VIP, the Wanfang Science and Technology Journal Full-text Database, the Chinese Biomedical Literature Search Database (CBM), PubMed, Embase, and the Cochrane Library. The search period was from database establishment to October 2021. The English literature was searched using Medical Subject Headings (MeSH) vocabulary combined with free words, such as 'breast augmentation' or 'breast prostheses', 'smooth implant' or 'textured implant', and 'capsular contracture'.

\section{Literature screening}

\section{Inclusion criteria}

The inclusion criteria were as follows: (I) a clinical trial comparing smooth and textured breast prostheses; (II) a 
detailed description of the study method, prosthesis type, and number of cases; (III) provision of data on the incidence of capsular contracture or raw data that could be used for calculation.

\section{Exclusion criteria}

The exclusion criteria were as follows: (I) basic research such as animal experiments; (II) reviews or meta-analyses; (III) literature that was re-published with the same data; (IV) literature from which data could not be obtained.

\section{Risk of bias evaluation and quality assessment}

Relevant criteria in the Jadad scale were used to evaluate the publication bias of randomized controlled trials, with a full score of 7 for the 4 items, where 1-3 indicated low quality and 4-7 indicated high quality. The Newcastle-Ottawa Score (NOS) scale was used to evaluate case-control studies and cohort studies, with a full score of 9 for the 8 items, where $>5$ indicated low quality articles and $<5$ indicated high quality articles.

\section{Data extraction}

After the literature was retrieved, Endnote X9 (Clarivate, Philadelphia, PA, USA) was used to excluded repeat literature, and 2 reviewers independently screened the articles. Unqualified articles were excluded based on the title and abstract. Once the original text and data were obtained, the full text was read. Data extraction was performed according to the inclusion and exclusion criteria. The extracted contents included the following: (I) the basic information of the article (title, author, publication date, and contact address); and (II) the basic characteristics of the study (study method, number of prostheses, and number of capsular contractures). In the case of any dispute between the data extraction and the quality evaluation, 2 persons negotiated or solved the difference of opinion with a third reviewer.

\section{Handling of data loss}

If the data couldn't be obtained by reading the original text, but the address link of the data could be found in the text, the required data was obtained by the link. If there was no data and no link, the original author was contacted to obtain the required data. If the author couldn't be contacted, the literature was excluded.

\section{Statistical analysis}

Stata 15.1 (StataCorp LLC, College Station, TX, USA) was used to combine the incidence rates and perform a metaanalysis to generate a forest plot. $\mathrm{I}^{2}$ was used to evaluate the heterogeneity among the included studies. If the $\mathrm{I}^{2}$ value was $<50 \%$, a fixed-effects model was used. If the $\mathrm{I}^{2}$ value was $>50 \%$, a random-effects model was used to combine the relevant study results. The source of heterogeneity was analyzed using a subgroup sensitivity analysis. Publication bias was assessed using a funnel plot test. The OR (odds ratio) was used as the analysis statistic to estimate the incidence of capsular contracture, which was expressed by the $95 \%$ CI. A P value of $<0.05$ indicated that the difference was statistically significant.

\section{Results}

\section{Literature search results and screening process}

A total of 728 articles were retrieved from the databases using the above search expressions. Of these, 335 articles were excluded and 393 were retained after duplicate screening with Endnote X9. A further 355 articles were excluded after preliminary screening by reading the titles and abstracts. Of the 38 remaining articles, 6 were not obtainable and were removed. After full-text screening, 19 articles were removed due to a lack of data or outcome, while 13 remained. Figure 1 shows the literature search results and screening process.

\section{Basic characteristics of the included articles}

A total of 13 articles met the inclusion criteria. The basic characteristics of these articles are shown in Table 1.

\section{Meta-analysis results}

Capsular contracture rate after breast augmentation with textured and smooth breast prostheses

A total of 13 studies (1,3-14) provided data on the incidence of capsular contracture after breast augmentation with both textured and smooth breast prostheses. The results of the heterogeneity test $\left(\mathrm{P}=0.020 ; \mathrm{I}^{2}=96 \%\right)$ using a randomeffects model is shown in Figure 2.

\section{Sensitivity analysis}

A further sensitivity analysis revealed that the study by Calobrace et al. (6) was a source of significant heterogeneity, as shown in Figure 3. 


\section{Identification of studies via databases and registers}
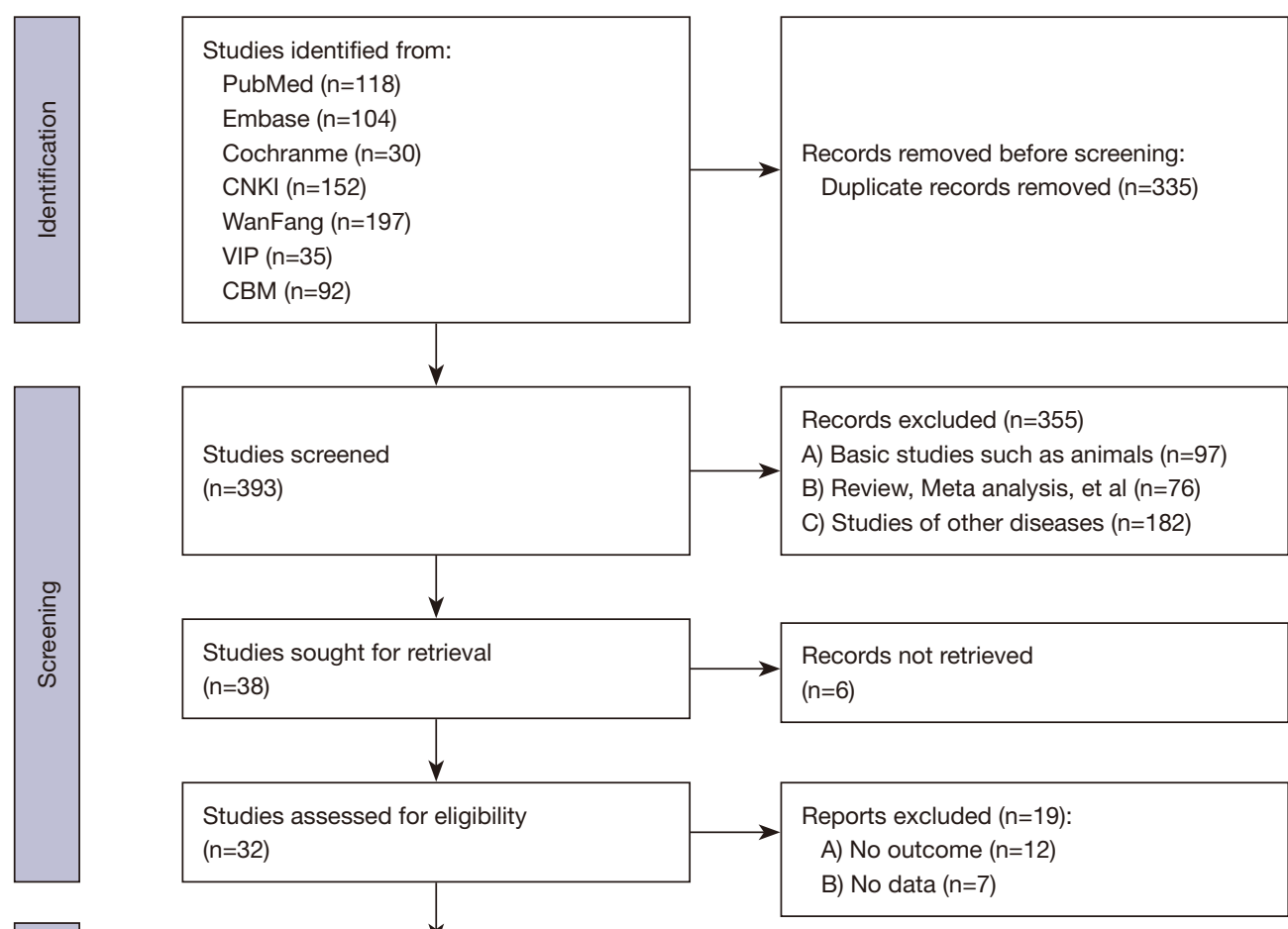

Studies included in meta-analysis $(n=13)$

Figure 1 Search and selection chart.

Table 1 Basic characteristics of the included articles

\begin{tabular}{|c|c|c|c|c|c|}
\hline Study & Year & Processing method & Case load & Membrane contracture number & Quality grade score \\
\hline Asplund (3) & 1996 & $\mathrm{Ss} / \mathrm{Ts}$ & $58 / 52$ & $5 / 8$ & 3 \\
\hline Burkhardt (4) & 1994 & $\mathrm{Ss} / \mathrm{Ts}$ & $45 / 45$ & $1 / 18$ & 4 \\
\hline Burkhardt (5) & 1995 & $\mathrm{Ss} / \mathrm{Ts}$ & $52 / 52$ & $7 / 12$ & 4 \\
\hline Calobrace (6) & 2018 & $\mathrm{Ss} / \mathrm{Ts}$ & $1,954 / 3,168$ & $124 / 475$ & 5 \\
\hline Fagrell (9) & 2001 & $\mathrm{Ss} / \mathrm{Ts}$ & $18 / 18$ & $1 / 4$ & 3 \\
\hline Hakelius (10) & 1992 & $\mathrm{Ss} / \mathrm{Ts}$ & $25 / 25$ & $0 / 11$ & 4 \\
\hline Handel (1) & 2006 & $\mathrm{Ss} / \mathrm{Ts}$ & $265 / 1,138$ & $9 / 44$ & 7 \\
\hline Liu (11) & 2016 & $\mathrm{Ss} / \mathrm{Ts}$ & $110 / 54$ & $2 / 2$ & 5 \\
\hline Wang (14) & 2003 & $\mathrm{Ss} / \mathrm{Ts}$ & $344 / 508$ & $9 / 13$ & 2 \\
\hline
\end{tabular}

Ss, smooth surface; Ts, textured surface. 


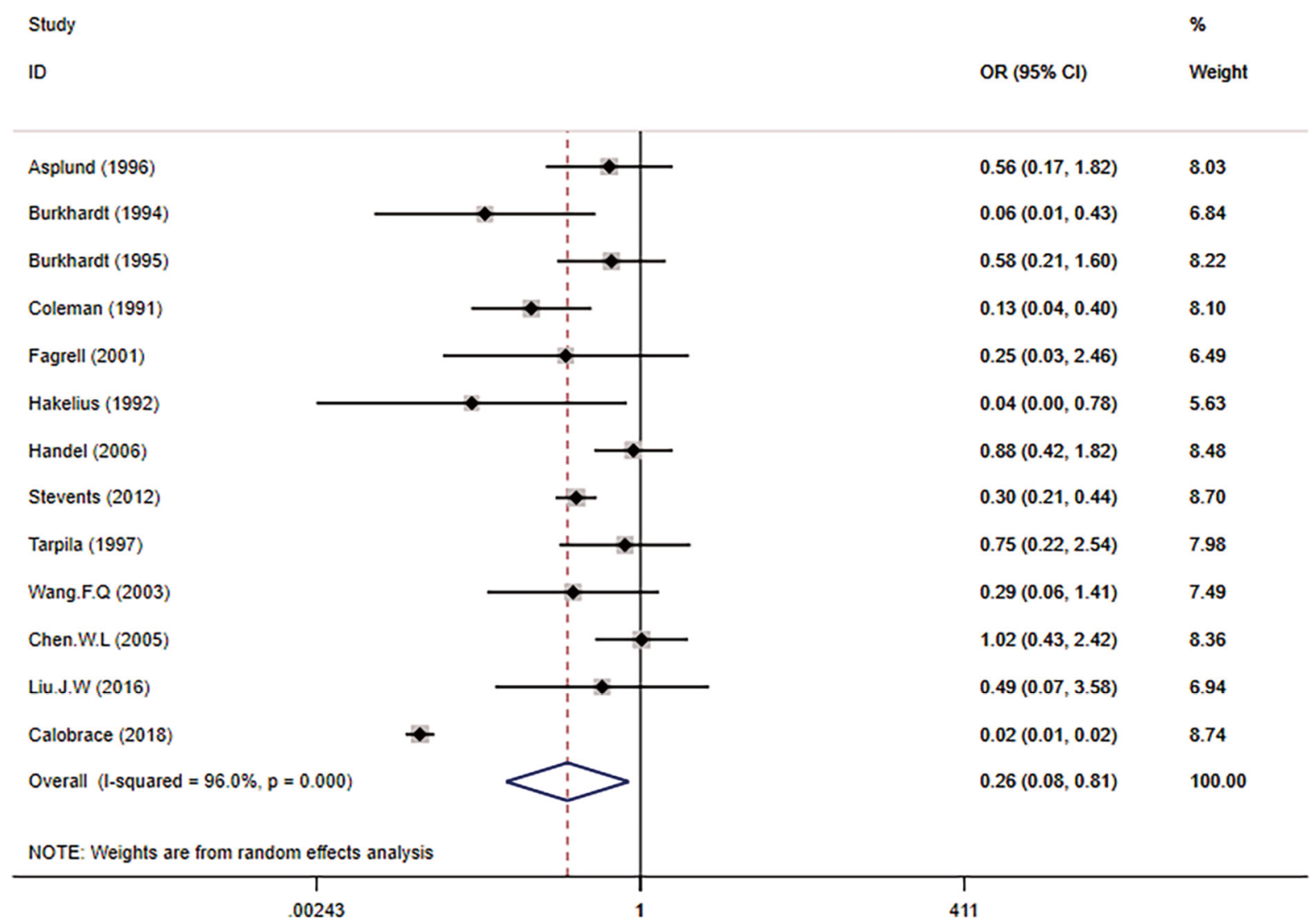

Figure 2 Forest plot of the combined results of capsular contracture rate.

\section{Capsular contracture rate after breast augmentation} with textured and smooth breast prostheses (sensitivity analysis)

After removal of the Calobrace et al. study, the results showed mild heterogeneity $\left(\mathrm{P}<0.001 ; \mathrm{I}^{2}=52.7 \%\right)$. As the study baseline was similar between the 2 groups and there was some clinical homogeneity, a random-effects model was selected for the analysis. The results suggested that the incidence rate of capsular contracture after breast augmentation with textured breast prosthesis was lower than that with smooth breast prosthesis $(\mathrm{OR}=0.37 ; 95 \%$ CI: $0.29,0.48)$. This was consistent with the results before removal, as shown in Figure 4.

\section{Subgroup analysis}

A subgroup analysis according to submammary augmentation and subpectoral augmentation showed that subpectoral augmentation (OR $=0.69 ; 95 \% \mathrm{CI}: 0.36,1.34$; $\left.\mathrm{P}=2.73 ; \mathrm{I}^{2}=4.7 \%\right)$. There was no significant difference in the data, suggesting that the incidence of capsular contracture after breast augmentation with subpectoral breast augmentation time surface breast prosthesis and hair surface breast prosthesis is similar; submammary breast augmentation $\left(\mathrm{I}^{2}=53.9 \%\right)$, suggesting that the heterogeneity was from submammary augmentation. As the study baseline was similar between the 2 groups and there was some clinical homogeneity, a random-effects model was selected for analysis ( $\mathrm{OR}=0.24 ; 95 \% \mathrm{CI}: 0.10$, $0.61 ; \mathrm{P}=0.03)$. The results suggested that for submammary augmentation, the incidence of capsular contracture was lower with textured prostheses than with smooth breast prostheses (Figure 5).

Funnel plot analysis of capsular contracture rate after breast augmentation with textured breast prosthesis and smooth breast prosthesis

Stata 15.1 was used to draw a funnel plot of the capsular contracture rate. The funnel plot results showed that the scatter points were asymmetric left and right, suggesting that there may be publication bias, as shown in Figure 6 . 


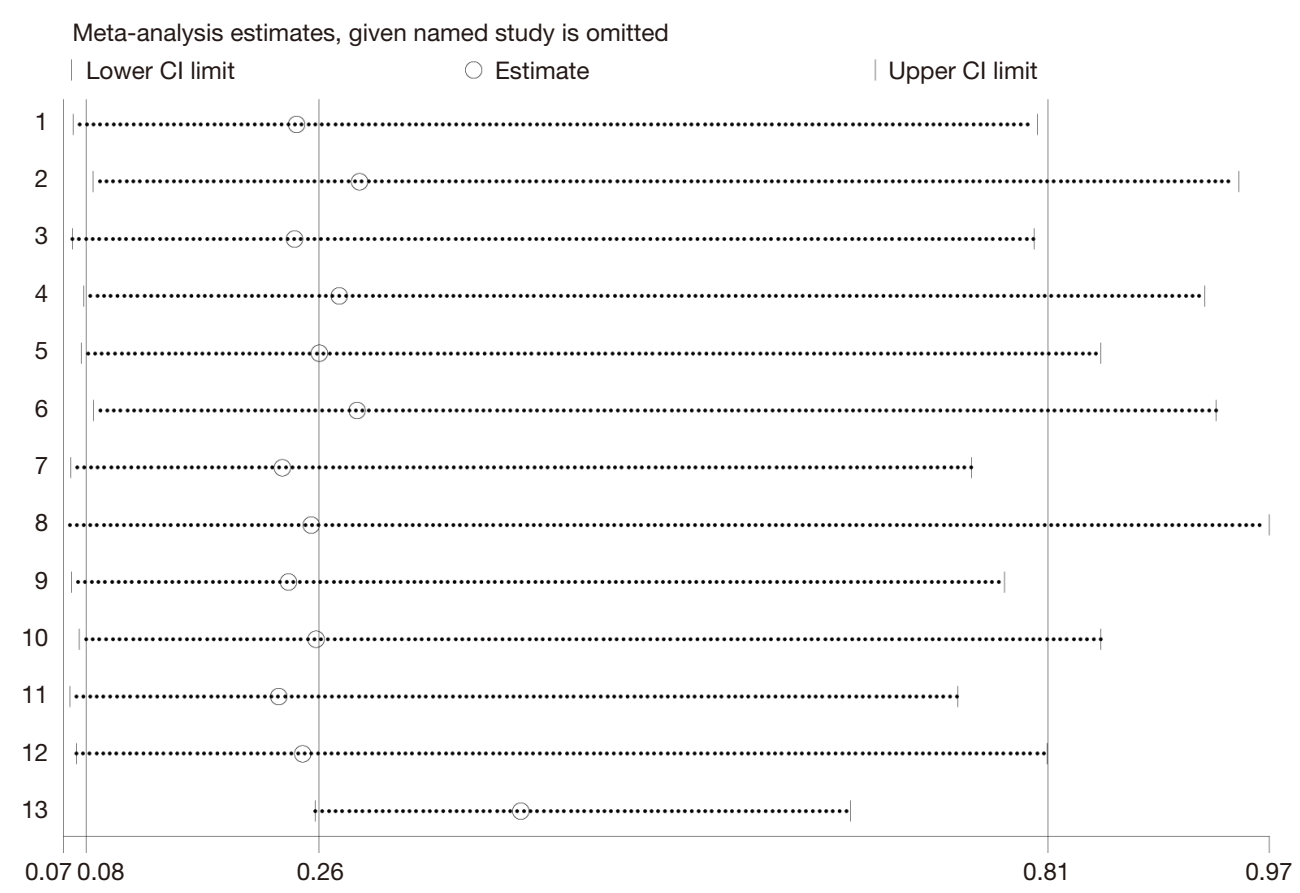

Figure 3 Sensitivity analysis.

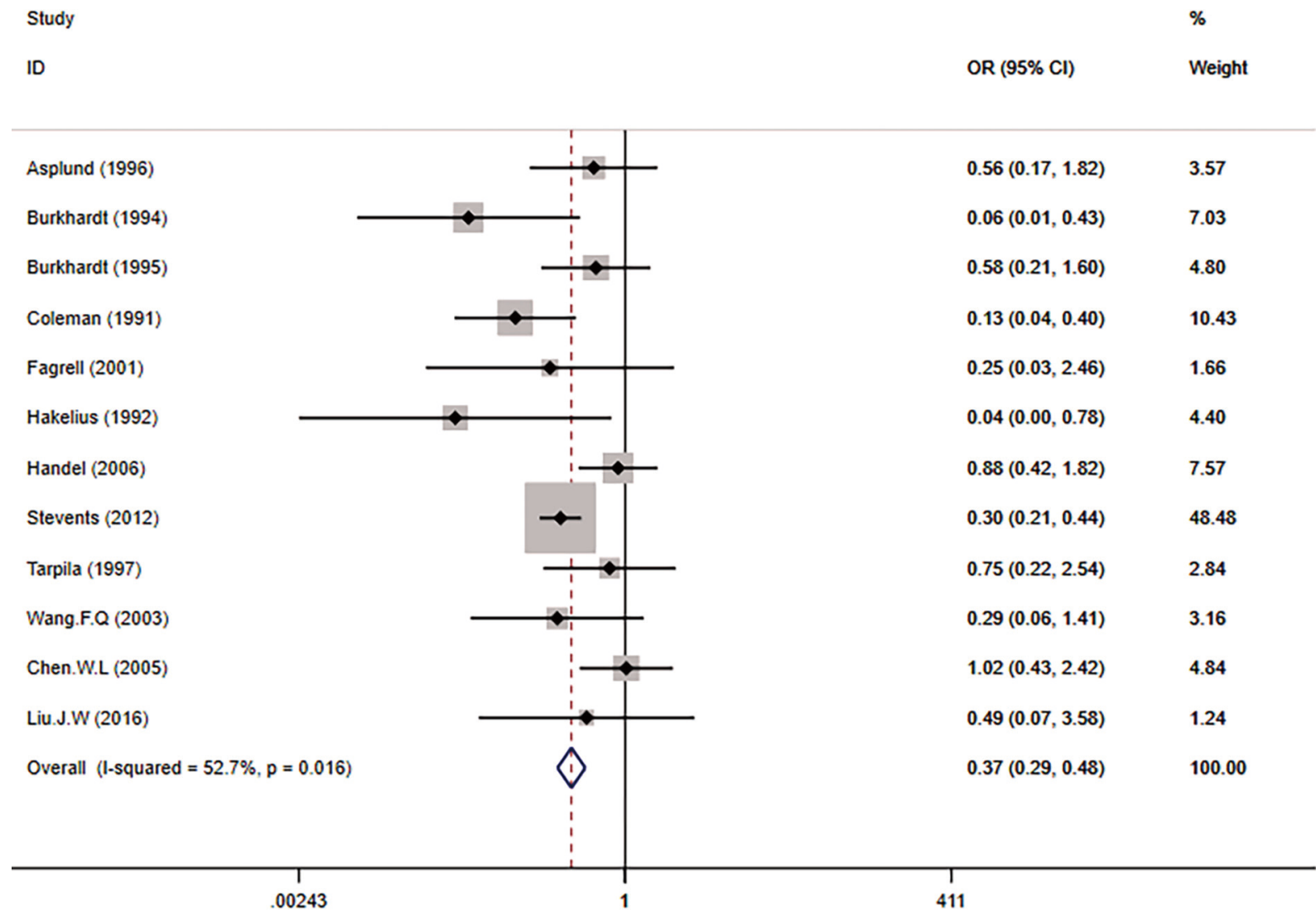

Figure 4 Forest plot of combined capsular contracture rate results (sensitivity analysis). 


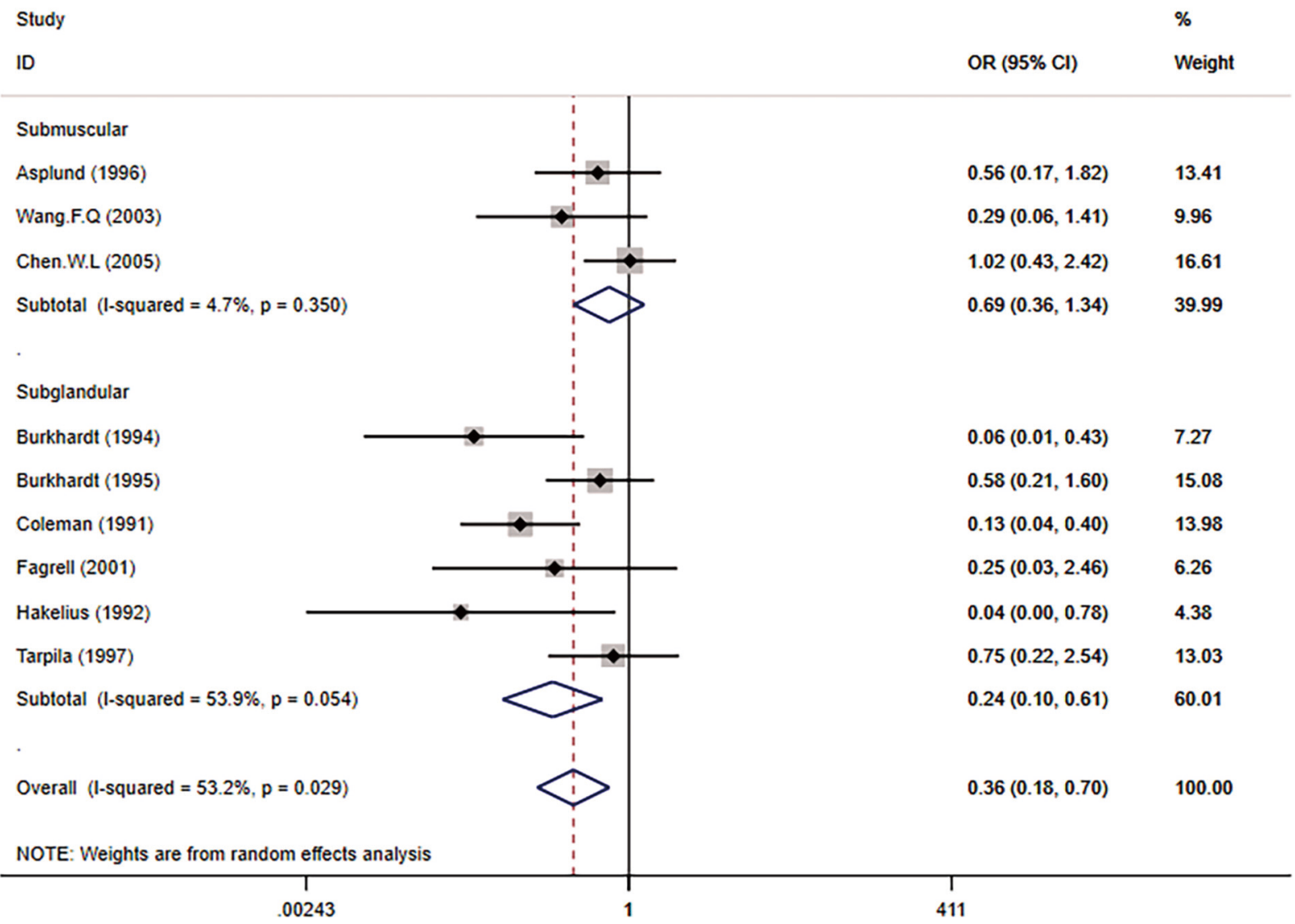

Figure 5 Subgroup analysis.

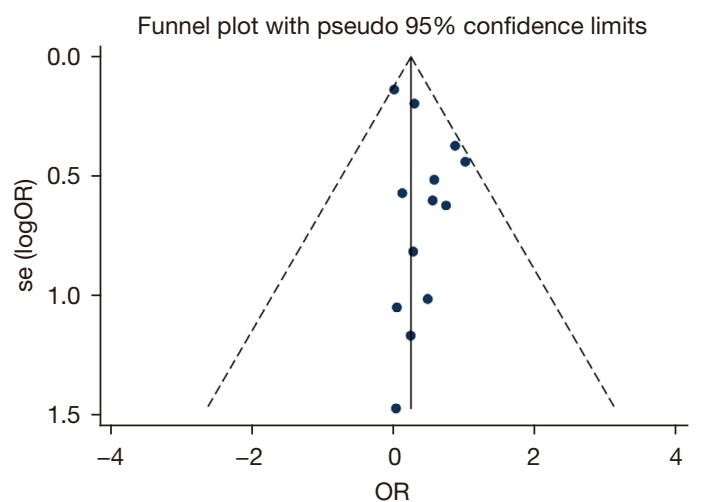

Figure 6 Funnel plot of capsular contracture rate after breast augmentation with textured and smooth breast prostheses.

\section{Discussion}

Meta-analysis is a method for re-analyzing results. By combining the results of multiple studies with the same purpose, meta-analysis can increase the sample size and improve the statistical power of the test, thus providing clearer results. This is particularly useful when the results of multiple studies are inconsistent or the conclusion is not statistically significant. Meta-analysis can employ either a fixed-effects model or a random-effects model, with the appropriate method selected according to the extent of heterogeneity between the studies.

Capsular contracture is a common complication following breast augmentation with prosthesis, with an incidence rate from $0 \%$ to $70 \%$ (15). This complication is the main cause of negative effects after breast augmentation, including breast hardening, breast distortion, and pain (16). In addition to the quality and type of the prosthesis itself, the occurrence of capsular contracture is related to the following factors: (I) residual foreign body; (II) infection; (III) incomplete postoperative drainage; (IV) too small a range of the separated cavities during surgery; and (V) the type of placement plane and incision (17). Correspondingly, the establishment of a reasonable surgical plan before surgery, the appropriate selection of prosthesis type and plane of 
placement, the employment of a strict and standardized operation and aseptic concept during surgery, and careful nursing after surgery with massage assistance (15) can effectively counteract these adverse factors and greatly reduce the incidence of capsular contracture. The mechanism of capsular contracture is not yet fully understood, but there are 2 main hypotheses: scar proliferation and infection (18). In recent years, many studies have shown that textured breast prostheses have a positive effect in reducing the incidence of capsular contracture after breast augmentation. However, some scholars believe that there is no difference in the capsular contracture rate between the 2 prostheses. We carried out this study to investigate this issue.

A total of 13 articles were included in the study, with a total sample size of 5,440 . All studies investigated the rate of capsular contracture of textured breast prosthesis and smooth breast prosthesis following augmentation mammaplasty. After combining the results, the meta-analysis found that the capsular contracture rate of smooth breast prosthesis after augmentation mammaplasty was significantly higher than that of textured breast prosthesis. This is consistent with the results of previous studies $(19,20)$. Since the included studies were not all randomized controlled trials and the study quality was uneven, some heterogeneity was present. A sensitivity analysis was performed and articles with significant heterogeneity were removed. The results were consistent with those before the removal. To further reduce bias, we performed a corresponding subgroup analysis. The results showed that the results of submammary augmentation were consistent with the overall results, but there was no significant difference in the results of subpectoral augmentation. The reason for this may be that the prosthesis was deeply located during subpectoral augmentation and not easily palpable even with capsular contracture, which would result in some errors while performing the Baker classification of capsular contracture.

This study has the following limitations: (I) the number of studies included was small; (II) the quality of the literature was uneven; (III) the included studies were not all randomized controlled trials, resulting in a degree of heterogeneity. In the future, relevant studies of higher quality and with a larger sample size should be included to further confirm the conclusion.

\section{Conclusions}

In summary, the capsular contracture rate after breast augmentation with textured breast prostheses was lower than that with smooth breast prostheses. Textured breast prostheses could significantly reduce the capsular contracture rate during breast augmentation in the submammary plane.

\section{Acknowledgments}

Funding: This project was supported by Hainan Province Clinical Medical Center.

\section{Footnote}

Reporting Checklist: The authors have completed the PRISMA reporting checklist. Available at https:// gs.amegroups.com/article/view/10.21037/gs-21-835/rc

Conflicts of Interest: All authors have completed the ICMJE uniform disclosure form (available at https://gs.amegroups. com/article/view/10.21037/gs-21-835/coif). The authors have no conflicts of interest to declare.

Ethical Statement: The authors are accountable for all aspects of the work in ensuring that questions related to the accuracy or integrity of any part of the work are appropriately investigated and resolved.

Open Access Statement: This is an Open Access article distributed in accordance with the Creative Commons Attribution-NonCommercial-NoDerivs 4.0 International License (CC BY-NC-ND 4.0), which permits the noncommercial replication and distribution of the article with the strict proviso that no changes or edits are made and the original work is properly cited (including links to both the formal publication through the relevant DOI and the license). See: https://creativecommons.org/licenses/by-nc-nd/4.0/.

\section{References}

1. Handel N, Cordray T, Gutierrez J, et al. A long-term study of outcomes, complications, and patient satisfaction with breast implants. Plast Reconstr Surg 2006;117:75767; discussion 768-72.

2. Cheng L, Jia Z. Evolution and Progress of Breast. Medicine and Philosophy (B) 2014;4:94-7.

3. Asplund O, Gylbert L, Jurell G, et al. Textured or smooth implants for submuscular breast augmentation: a controlled study. Plast Reconstr Surg 1996;97:1200-6.

4. Burkhardt BR, Demas CP. The effect of Siltex texturing 
and povidone-iodine irrigation on capsular contracture around saline inflatable breast implants. Plast Reconstr Surg 1994;93:123-8; discussion 129-30.

5. Burkhardt BR, Eades E. The effect of Biocell texturing and povidone-iodine irrigation on capsular contracture around saline-inflatable breast implants. Plast Reconstr Surg 1995;96:1317-25.

6. Calobrace MB, Stevens WG, Capizzi PJ, et al. Risk Factor Analysis for Capsular Contracture: A 10-Year Sientra Study Using Round, Smooth, and Textured Implants for Breast Augmentation. Plast Reconstr Surg 2018;141:20S-8S.

7. Chen WL, Li QF, Lei H, et al. The clinic analysis of complications of varied breast implant. Zhonghua Zheng Xing Wai Ke Za Zhi 2005;21:172-4.

8. Coleman DJ, Foo IT, Sharpe DT. Textured or smooth implants for breast augmentation? A prospective controlled trial. Br J Plast Surg 1991;44:444-8.

9. Fagrell D, Berggren A, Tarpila E. Capsular contracture around saline-filled fine textured and smooth mammary implants: a prospective 7.5-year follow-up. Plast Reconstr Surg 2001;108:2108-12; discussion 2113.

10. Hakelius L, Ohlsén L. A clinical comparison of the tendency to capsular contracture between smooth and textured gel-filled silicone mammary implants. Plast Reconstr Surg 1992;90:247-54.

11. Liu J, Zhang W, Zhu B. Clinical review of breast prosthesis and hierarchy affecting envelope clonture. Chinese Beauty Medicine 2016;25:11-4.

Cite this article as: Zhai $\mathrm{P}, \mathrm{Wu} \mathrm{Y}$, Yang D, Ma W, Zhai Y, Xie H. A systematic review and meta-analysis of capsular contracture rate after breast augmentation with textured and smooth breast prostheses. Gland Surg 2022;11(1):166-174. doi: $10.21037 /$ gs-21-835
12. Stevens WG, Harrington J, Alizadeh K, et al. Five-year follow-up data from the U.S. clinical trial for Sientra's U.S. Food and Drug Administration-approved Silimed® brand round and shaped implants with high-strength silicone gel. Plast Reconstr Surg 2012;130:973-81.

13. Tarpila E, Ghassemifar R, Fagrell D, et al. Capsular contracture with textured versus smooth saline-filled implants for breast augmentation: a prospective clinical study. Plast Reconstr Surg 1997;99:1934-9.

14. Wang SQ. Prospective study of fiber-coated contractures in hairy and silicone gel prosthesis augmentation. Sichuan Medicine 2003;1:5.

15. Liu Y. Histological response of the in silico gel prosthesis. Railway Medicine 1999;5:353-5.

16. Chen Z, Luo D, Shen W. Progress in drug envelope control of fiber contracture after prosthetic breast augmentation. Chongqing Medicine 2004;7:1109-10.

17. Wiener TC. Relationship of incision choice to capsular contracture. Aesthetic Plast Surg 2008;32:303-6.

18. Rohrich RJ, Kenkel JM, Adams WP. Preventing capsular contracture in breast augmentation: in search of the Holy Grail. Plast Reconstr Surg 1999;103:1759-60.

19. Camirand A, Doucet J. Breast augmentation: teaching our patients how compression can help prevent capsular contracture. Aesthetic Plast Surg 2000;24:221-6.

20. Li S. Meta analysis of envelope contracture rate after hairy and light breast prosthesis augmentation. Mentor: Tan Qian. Nanjin university, 2014. 